\title{
SIMULTANEOUS HEAVY ION DISSOCIATION AT ULTRARELATIVISTIC ENERGIES
}

\author{
I.A. PSHENICHNOV ${ }^{1,2 a}$, J.P. BONDORF ${ }^{3}$, S. MASETTI ${ }^{2}$, \\ I.N. MISHUSTIN ${ }^{3,4}$, A. VENTURA ${ }^{2}$ \\ 1 Institute for Nuclear Research, Russian Academy of Science, \\ 117312 Moscow, Russia \\ 2 Italian National Agency for New Technologies, \\ Energy and Environment, 40129 Bologna, Italy \\ ${ }^{3}$ Niels Bohr Institute, DK-2100 Copenhagen, Denmark \\ ${ }^{4}$ Kurchatov Institute, Russian Research Center, \\ 123182 Moscow, Russia
}

We study the simultaneous dissociation of heavy ultrarelativistic nuclei followed by the forward-backward neutron emission in peripheral collisions at colliders. The main contribution to this particular heavy-ion dissociation process, which can be used as a beam luminosity monitor, is expected to be due to the electromagnetic interaction. The Weizsäcker-Williams method is extended to the case of simultaneous excitation of collision partners which is simulated by the RELDIS code. A contribution to the dissociation cross section due to grazing nuclear interactions is estimated within the abrasion model and found to be relatively small.

\section{Single and mutual electromagnetic dissociation}

Let us consider a collision of heavy ultrarelativistic nuclei with the masses and charges $\left(A_{1}, Z_{1}\right)$ and $\left(A_{2}, Z_{2}\right)$ at the impact parameter $b$ exceeding the sum of nuclear radii, $b>R_{1}+R_{2}$. According to the Weizsäcker-Williams (WW) method, the impact of the Lorentz-boosted Coulomb field of the nucleus $A$ on the collision partner $A_{2}$ is treated as the absorption of equivalent photons 1 . The mean number of photons absorbed by the nucleus $A_{2}$ in such collision is:

$$
m_{A_{2}}(b)=\int N_{Z_{1}}\left(E_{1}, b\right) \sigma_{A_{2}}\left(E_{1}\right) d E_{1},
$$

where the spectrum of virtual photons, $N_{Z_{1}}\left(E_{1}, b\right)$, and the total photoabsorption cross section, $\sigma_{A_{2}}\left(E_{1}\right)$, are used.

Assuming that the probability of multiphoton absorption is given by the Poisson distribution with the mean multiplicity $m_{A_{2}}(b)$, one has the cross section for the single electromagnetic dissociation to a given channel $i$ :

$$
\sigma_{1}(i)=2 \pi \int_{b_{c}}^{\infty} b d b P_{A_{1}}(b), b_{c}=1.34\left(A_{1}^{1 / 3}+A_{2}^{1 / 3}-0.75\left(A_{1}^{-1 / 3}+A_{2}^{-1 / 3}\right)\right),
$$

${ }^{a}$ e-mail: pshenichnov@nbi.dk 


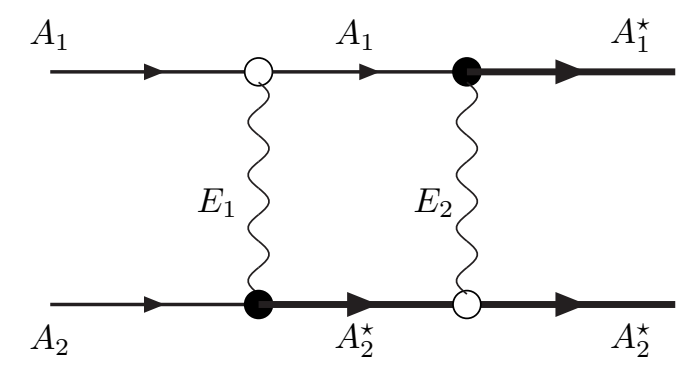

Figure 1: Mutual electromagnetic dissociation of relativistic nuclei. The open and closed circles denote the elastic and inelastic vertices, respectively.

where $b_{c}$ is in fm and the probability of dissociation at impact parameter $b$ is given by:

$$
P_{A_{1}}(b)=e^{-m_{A_{1}}(b)} \int d E_{1} N_{Z_{1}}\left(E_{1}, b\right) \sigma_{A_{2}}\left(E_{1}\right) f_{A_{2}}\left(E_{1}, i\right) .
$$

Here $f_{A_{2}}\left(E_{1}, i\right)$ is the branching ratio for the considered channel $i$ in the absorption of a photon with the energy $E_{1}$ on the nucleus $A_{2}$. These values are calculated by photonuclear reaction models 2.3 or taken from experiments.

In the WW method, the graph for the mutual electromagnetic dissociation, Fig. 1, may be constructed from two graphs of the single dissociation by interchanging the roles of "emitter" and "absorber" at the secondary photon exchange. Such procedure is possible since the first emitted photon with $E_{1} \leq E_{\max } \sim \gamma / R$ does not change essentially the total energy, $E_{A}=\gamma M_{A}$, of the emitting nucleus, $E_{\max } / E_{A} \approx 1 / R M_{A} \sim 10^{-4}$, and there are no correlations between the energies $E_{1}$ and $E_{2}$. In other words, both photon exchanges may be considered as independent processes. Moreover, at ultrarelativistic energies the collision time is much shorter then a typical deexcitation period when a nucleus loses its charge via the proton emission or fission. It means that the equivalent photon spectrum from the excited nucleus, $A_{2}^{\star}$, is equal to the spectrum from the nucleus in its ground state, $A_{2}$, see Fig. [1.

Therefore the cross section for the mutual electromagnetic dissociation of the nuclei $A_{1}$ and $A_{2}$ to given channels $i$ and $j$ is given by:

$$
\sigma_{m}(i, j)=2 \pi \int_{b_{c}}^{\infty} b d b P_{A_{1}}(b) P_{A_{2}}(b) .
$$


Substituting $P_{A}(b)$ for each of the nuclei one has:

$$
\sigma_{m}(i, j)=\iint d E_{1} d E_{2} \mathcal{N}_{m}\left(E_{1}, E_{2}\right) \sigma_{A_{2}}\left(E_{1}\right) \sigma_{A_{1}}\left(E_{2}\right) f_{A_{2}}\left(E_{1}, i\right) f_{A_{1}}\left(E_{2}, j\right),
$$

with the spectral function $\mathcal{N}_{m}\left(E_{1}, E_{2}\right)$ defined for the mutual dissociation:

$$
\mathcal{N}_{m}\left(E_{1}, E_{2}\right)=2 \pi \int_{b_{c}}^{\infty} b d b e^{-2 m(b)} N_{Z_{1}}\left(E_{1}, b\right) N_{Z_{2}}\left(E_{2}, b\right)
$$

\section{Nucleon removal in grazing nuclear collisions}

The cross section for the abrasion of $n$ neutrons and $z$ protons from the projectile $\left(A_{1}, Z_{1}\right)$ in a peripheral collision with the farget $\left(A_{2}, Z_{2}\right)$ may be derived from the Glauber multiple scattering theory $\omega^{-}$. A simple parameterization exists for the single neutron removal cross section 5 :

$$
\sigma_{n u c}(1 n)=\frac{A_{1}-Z_{1}}{A_{1}} \sigma_{G} P_{e s c}, \quad \sigma_{G}=2 \pi\left(b_{c}-\frac{\Delta b}{2}\right) \Delta b,
$$

where $\Delta b \approx 0.5 \mathrm{fm}$ and $P_{\text {esc }} \approx 0.75$ is the probability for a neutron to escape without suffering the interaction with a spectator fragment. This can be extended to the case of mutual $(1 \mathrm{n}, 1 \mathrm{n})$ emission:

$$
\sigma_{n u c}(1 n, 1 n)=\left(\frac{A_{1}-Z_{1}}{A_{1}}\right)\left(\frac{A_{2}-Z_{2}}{A_{2}}\right) \sigma_{G} P_{e s c}^{2} .
$$

\section{Results and discussion}

The results of the abrasion model for the charge changing cross sections of the single dissociation of $158 \mathrm{~A} \mathrm{GeV}{ }^{208} \mathrm{~Pb}$ ions are shown in Fig. 2 2 in addition to the electromagnetic contribution calculated by the RELDIS code. As one can see, the electromagnetic contribution dominates for few nucleon removal process. The interaction of knocked out nucleons with spectators and spectator de-excitation process itself were neglected in this version of the abrasion model. However, good agreement with the experimental datal is found.

After this verification the model can be extrapolated to the energies of RHIC and LHC heavy-ion colliders. There is a proposal to use the simultaneous neutron emission for beam luminosity monitoring via the correlated registration of forward neutrons in zero degree calorimeters at RHIC $\mathrm{Z}$. The model predicts $\sigma_{m}(1 n, 1 n) \approx 750$ and $970 \mathrm{mb}$ for $\mathrm{AuAu}$ and $\mathrm{PbPb}$ collisions at RHIC and LHC, respectively, for the correlated single neutron emission. 


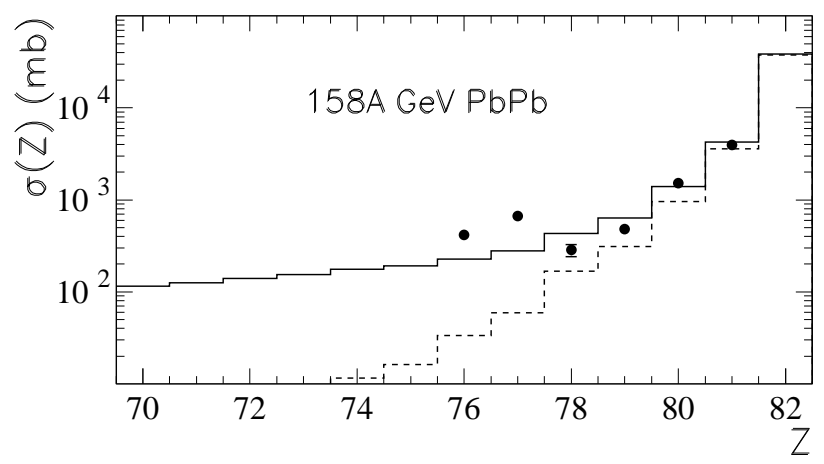

Figure 2: Charge changing cross sections of ${ }^{208} \mathrm{~Pb}$ ions at CERN SPS energies. The solid and dashed-line histograms are the RELDIS code results with and vithout contribution of the abrasion process, respectively. The points are experimental data $\mathrm{O}$.

We found that an important part of the dissociation events leading to the single neutron emission is accompanied by the emission of charged particles and nuclear fragments. Such events are due to the equivalent photon absorption above the Giant Resonance region. For grazing nuclear collisions Eq. (7) gives $\sigma_{n u c}(1 n, 1 n) \approx 100 \mathrm{mb}$. Our detailed consideration of the mutual nuclear dissociation is in progress.

We are grateful to A.S. Botvina, G. Dellacasa, J.J. Gaardhøje, G. Giacomelli, A.B. Kurepin and S. White for useful discussions. I.A.P. and I.N.M. are indebted to the Organizing Committee of Bologna 2000 Conference for the kind hospitality and financial support.

\section{References}

1. I.A. Pshenichnov et al., Phys. Rev. C60, 044901 (1999) and references therein.

2. M.B. Chadwick et al., Acta Phys. Slov. 45, 633 (1995).

3. A.S. Iljinov et al., Nucl. Phys. A616, 575 (1997).

4. J. Hüfner et al., Phys. Rev. C12, 1888 (1975).

5. C.J. Benesh et al., Phys. Rev. C40, 1198 (1989).

6. H. Dekhissi et al., Nucl. Phys. A662, 207 (2000).

7. A.J. Baltz et al., Nucl. Instrum. Methods A417, 1 (1998). 